Relations industrielles

Industrial Relations

\title{
Index - Volume 16 - 1961
}

Volume 16, numéro 4, octobre 1961

URI : https://id.erudit.org/iderudit/1021705ar

DOI : https://doi.org/10.7202/1021705ar

Aller au sommaire du numéro

Éditeur(s)

Département des relations industrielles de l’Université Laval

\section{ISSN}

0034-379X (imprimé)

1703-8138 (numérique)

Découvrir la revue

Citer ce document

(1961). Index - Volume 16 - 1961. Relations industrielles / Industrial Relations,

16(4), 526-532. https://doi.org/10.7202/1021705ar

Tous droits réservés (C Département des relations industrielles de l’Université Laval, 1961
Ce document est protégé par la loi sur le droit d'auteur. L'utilisation des services d'Érudit (y compris la reproduction) est assujettie à sa politique d'utilisation que vous pouvez consulter en ligne.

https://apropos.erudit.org/fr/usagers/politique-dutilisation/ 


\section{IN DEX \\ VOLUME $16-1961$}

Assurance chômage, Séparer le Service National de placement et la Commission d', (no 2), pp. 264-267.

Authority in the Enterprise, Property and, Gérard Dion, (no 1), pp. 30-48.

Autorité dans l'entreprise, La propriété et le fondement de l', Gérard Dion, (no 1), p. 48.

Canadian Social Security: Problems and Perspectives, Claude Morin, (no 1), p. 25.

Canadienne: problèmes et perspectives, La sécurité sociale, Claude Morin, (no 1), pp. 3-25.

Centrales syndicales et le problème du chômage, Les, Jacques St-Laurent, (no 2), pp. 238-242.

Chômage, Le, (Mémoire de la C.S.N. au cabinet fédéral) (no 2), pp. 259-262.

Chômage et économie de services, Claude Morin, (no 3), pp. 340-343.

Chômage, Les centrales syndicales et le problème du, Jacques St-Laurent, (no 2), pp. 238-242.

Chômage pour le salarié du Québec, Conséquences du, Gérald Fortin, M.-Adélard Tremblay et Marc Laplante, (no 3), pp. 289-310.

Comités paritaires, organismes originaux et nécessaires, Les, Raymond Gérin, (no 1), pp. 53-58.

Committees, Original and Indispensable Institutions, Parity, Raymond Gérin, (no 1), p. 58.

Commission d'assurance chômage, Séparer le Service National de placement et la, (no 2), pp. 264-267.

Confederation of National Trade Unions, Statement of Principles of the, (no 1), pp. 118-123.

Confédération des Syndicats Nationaux, Déclaration de principes de la, (no 1), pp. 113-118.

Consequences of Unemployment, Gérald Fortin, M.-Adélard Tremblay et Marc Laplante, (no 3), pp. 310-315.

Continuité des opérations dans l'industrie de la pulpe et du papier, La, Jacques StLaurent, (no 2), pp. 242-247.

C.S.N. a quarante ans, La, Jean Marchand, (no 4), pp. 471-475.

C.S.N., Chronologie de l'évolution confessionnelle de la CTCC, (no 1), pp. 102-113.

C.S.N 、 L'unité syndicale et la, (no 2), pp. 267-269. 
CTCC (CSN), Chronologie de l'évolution confessionnelle de la, (no 1), pp. 102-113.

C.R.O., Analyse critique de la Loi des relations ouvrières et du règlement no 1 de la, Alfred Charpentier, (no 1), pp. 59-83.

C.R.O., Analyse critique de la Loi des relations ouvrières et du règlement no 1 de la, Alfred Charpentier, (no 2), pp. 206-238.

C.R.O. (III), Analyse critique de la Loi des relations ouvrières et du règlement no 1 de la, Alfred Charpentier, ( no 3), pp. 328-340.

Démocratie organique et l'Encyclique Mater et Magistra, La, Gérard Dion, (no 4), pp. 460-464.

Depressed Areas in Italy: Data and Commentary, The Industrialization of, G.B. Bozzola, (no 2), pp. 184-205.

Economie de services, Chômage et, Claude Morin, (no 3), pp. 340-343.

Employer, The Government as, J.C. Best, (no 2), pp. 160-182.

Employeur, Le gouvernement comme, J.C. Best, (no 2), p. 182.

Entreprise, La propriété et le fondement de lautorité dans l', Gérard Dion, (no 1), p. 48.

Enterprise, Property and Authority in the, Gérard Dion, (no 1), pp. 30-48.

Evaluation des tâches, L', Jean-Paul Deschênes, (no 2), pp. 145-159.

Evaluation, Job, Jean-Paul Deschênes, (no 2), p. 159.

Evolution économique et sociale, Réflexions chrétiennes en face de l', (no 2), pp. 262-264.

Gouvernement comme employeur, Le, J.C. Best, (no 2), p. 182.

Government as Employer, The, J.C. Best, (no 2), pp. 160-182.

Grève du rail: partie remise ou contremandée?, La, Léo Roback, (no 1), pp. 83-89.

Industrialisation dans les régions sous-développées d'Italie: les données et les commentaires, $L^{\prime}$, G.B. Bozzola, (no 2), p. 205.

Industrialization of Depressed Areas in Italy: Data and Commentary, The, G.B. Bozzola, (no 2), pp. 184-205.

Industrie de la pulpe et du papier, Continuité des opérations dans l', Jacques StLaurent, (no 2), pp. 242-247.

Industry Council Plan and « Mater et Magistra », Gérard Dion, (no 4), ppp. 443460.

Italy: Data and Commentary, The Industrialization of Deressed Areas in, G.B. Bozzola, (no 2), pp. 184-205.

Italie: les données et les commentaires, L'industrialisation dans les régions sousdéveloppées d', G.B. Bozzola, (no 2), p. 205.

Job Evaluation, Jean-Paul Deschênes, (no 2), p. 159. 
Juridictions syndicales, Les, Gérard Picard, (no 4), pp. 464-471.

Labour Law (1961), Some New Trends in Quebec, Roger Chartier, (no 4), pp. 419-427.

Législation du travail (1961), Le texte intégral des récents amendements d la, (no 4), pp. 498-502.

Législation québecoise du travail - (1961) - L'évolution de la, Roger Chartier, (no 4), pp. 381-419.

Loi des relations ouvrières et du règlement no 1 de la C.R.O., Analyse critique de la, Alfred Charpentier, (no 1), pp. 59-83.

Loi des relations ouvrières et du règlement no 1 de la C.R.O., Analyse critique de la, (no 2), pp. 206-238.

Loi des relations ouvrières et du règlement no 1 de la C.R.O. (III), Analyse critique de la, Alfred Charpentier, (no 3), pp. 328-340.

Management, Re-examination and Re-organization of Relations Between Workers and, A.G. Cardinal Cicognani, (no 4), ppp. 504-507.

Mater et Magistra, Démocratie organique et l'Encyclique, Gérard Dion, (no 4), pp. $460-464$.

Mater et Magistra, Industry Council Plan and, Gérard Dion, (no 4), pp. 443-460.

Papier, Continuité des opérations dans l'industrie de la pulpe et du, Jacques StLaurent, (no 2), pp. 242-247.

Paritaires, organismes originaux et nécessaires, Les comités, Raymond Gérin, (no 1), pp. 53-58.

Parity Committees, Original and Indispensable Institutions, Raymond Gérin, (no 1), p. 58.

Personnel Man, Some Unfinished Business in the Professionnalization of the, Oswald Hall, (no 3), pp. 315-323.

Personnel, un professionnel?, Le spécialiste du, Oswald Hall, (no 3), pp. 323-328.

Professionnel?, Le spécialiste du personnel, un, Oswald Hall, (no 3), pp. 323-328.

Property and Authority in the Enterprise, Gérard Dion, (no 1), pp. 30-48.

Propriété et le fondement de l'autorité dans l'entreprise, La, Gérard Dion, (no 1), p. 48.

Professionnal Organization: A Confused Notion, The, (no 4), pp. 439-443.

Professionnalization of the Personnel Man, Some Unfinished Business in the, Oswald Hall, (no 3), pp. 315-323.

Professionnelle et syndicalisme - essai de réévaluation en regard des exigences présentes, Organisation, Jean-Réal Cardin, (no 4), pp. 427-439.

Professionnel, L'enseignement technique et, (no 4), pp. 496-498.

Publications en relations industrielles, Claude Gaudreau, (no 2), pp. 247-250. 
Pulpe et du papier, Continuité des opérations dans l'industrie de la, Jacques StLaurent, (no 2), pp. 242-247.

Québecoise du travail - 1961 - L'évolution de la législation, Roger Chartier, (no 4), pp. 381-419.

Québec, Conséquences du chômage pour le salarié du, Gérald Fortin, M.-Adélard Tremblay et Marc Laplante, (no 3), pp. 289-310.

Quebec Labour Law (1961), Some New Trends in, Roger Chartier, (no 4), p. 419427.

Rail: partie remise ou contremandée?, La grève du, Léo Roback, (no 1), pp. 83-89.

Réflexions chrétiennes en face de l'évolution économique et sociale, (no 2), pp. 262 264.

Relations between Workers and Management - Re-examination and Re-organization of, A.G. Cardinal Cicognani, (no 4), pp. 504-507.

Relations ouvrières et du règlement no 1 de la C.R.O., Analyse critique de la Loi des, Alfred Charpentier, (no 1), pp. 59-83.

Relations ouvrières et du règlement no 1 de la C.R.O., Analyse critique de la Loi des, Alfred Charpentier, (no 2), pp. 206-238.

Relations ouvrières et du règlement no 1 de la C.R.O. (III), Analyse critique de la Loi des, Alfred Charpentier, (no 3), pp. 328-340.

Relations industrielles, Publications en, Claude Gaudreau, (no 2), pp. 247-250.

Salarié du Québec, Conséquences du chômage pour le, Gérald Fortin, M.-Adélard Tremblay et Marc Laplante, (no 3), pp. 289-310.

Service national de placement et la Commission d'assurance chômage, Séparer le, (no 2), pp. 264-267.

Sécurité sociale canadienne: problèmes et perspectives, La, Claude Morin, (no 1), pp. 3-25.

Social Security: Problems and Perspectives, Canadian, Claude Morin, (no 1), p. 25.

Syndicales, Les juridictions, Gérard Picard, (no 4), pp. 464-471.

Syndicales et le problème du chômage, Les centrales, Jacques St-Laurent, (no 2), pp. 238-242.

Syndicalisme - essai de réévaluation en regard des exigences présentes, Organisation professionnelle et, Jean-Réal Cardin, (no 4), pp. 427-439.

Tâches, L'évaluation des, Jean-Paul Deschênes, (no 2), pp. 145-159.

Technique et professionnel, L'enseignement, (no 4), pp. 496-498.

Travail - 1961 - Evolution de la législation québecoise du, Roger Chartier, (no 4), pp. 381-419.

Travail, Le texte intégral des récents amendements d̀ la législation du, (no 4), pp. 498-502. 
Unemployment, Consequences of, Gérald Fortin, M.-Adélard Tremblay et Marc Laplante, (no 3), pp. 310-315.

Unité syndicale et la C.S.N., L', (no 2), pp. 267-269.

Les Tribunaux du travail:

Composition et le système de procédure des tribunaux du travail, La, André Desgagné, (no 3), pp. 356-357.

Droit du travail, Le particularisme et l'esprit propre de, Jean-Réal Cardin (no 3), pp. 359-362.

Expériences étrangères, René Mankiewicz, (no 3), pp. 352-354.

Procédure des tribunaux du travail, La composition et le système de, André Desgagné, (no 3), pp. 356-357.

Système de procédure des tribunaux du travail, La composition et le, André Desgagné, (no 3), pp. 356-357.

Travail, Le particularisme et l'esprit propre de droit du, Jean-Réal Cardin, (no 3), pp. 359-362.

Tribunaux du travail, La composition et le système de procédure des, André Desgagné, (no 3), pp. 356-357.

Tribunaux du travail dans l'ensemble de l'organisation judiciaire, Place des, Marc Lapointe, (no 3), pp. 357-359.

Tribunaux du travail, Juridiction des, Gérard Picard, (no 3), pp. 354-356.

Tribunaux du travail, Nature et raisons d'être des, Emile Gosselin, (no 3), pp. $350-356$.

Tribunaux du travail: propos préliminaires, Les, Gérard Dion, (no 3), pp. 348350.

Workers and Management, Re-examination and Re-organization of Relations Between, A.G. Cardinal Cicognani, (no 4), pp. 504-507.

\section{JURISPRUDENCE DU TRAVAIL}

Agreement When a Plant Closes Down, Renewal of an, (no 1), pp. 100-101.

Ancienneté et mise d̀ pied, (no 1), pp. 89-93.

Appel à un tribunal d'arbitrage d'une décision de la C.R.O. - Absence de juridiction du tribunal d'arbitrage, (no 4), pp. 487-492.

Appointment, Openings and the Expected Permanency of, (no 2), pp. 253-255.

Arbitrage d'une décision de la C.R.O. - Absence de juridiction du tribunal d'arbitrage, Appel à un tribunal d', (no 4), pp. 487-492.

Arrêt de travail, Menace à la sécurité et légalité de l’, (no 2), pp. 255-259.

Avis de congé chez les commis de magasin, (no 3), pp. 343-344. 
Award, Trial Period Denied, Binding Authority of a Previous, (no 2), pp. 252-253.

Binding Authority of a Previous Award, Trial Period Denied, (no 2), pp. 252-253.

Collective Agreement, Disciplinary Measures Where Exists an Alleged Violation of $a$, (no 3), pp. 344-346.

Commis de magasin, Avis de congé chez les, (no 3), pp. 343-344.

Congédiement et procédures requises, (no 3), pp. 346-348.

Congédiement pour activités syndicales - Juridiction de la Commission de Relations ouvrières, (no 4), pp. 485-487.

Convention, Liquidation des affaires et renouvellement de, (no 1), pp. 101-102.

Damages Awarded by Arbitration Board - Application for Certiori to Quash Award - Whether Board has Power to Award and Assess Damages, (no 4), pp. 479-481.

Damages - Power of Board to Award, (no 4), pp. 475-479.

Disciplinary Measures Where Exists an Alleged Violation of a Collective Agreement, (no 3), pp. 344-346.

Employé mis à pied demeure-t-il un employé régulier?, L', (no 1), pp. 95-100.

Juridiction du tribunal d'arbitrage, Appel à un tribunal d'arbitrage d'une décision de la C.R.O., Absence de, (no 4), pp. 487-492.

Jurisdiction of a Labour Board, (no 1), pp. 93-95.

Labour Board, Jurisdiction of a, (no 1), pp. 93-95.

Menace à la sécurité et légalité de l'arrêt de travail, (no 2), pp. 255-259.

Mise à pied, Ancienneté et, (no 1), pp. 89-93.

Permanency of Appointment, Openings and the Expected, (no 2), pp. 253-255.

Reduction of the Working Force, Defined, (no 2), pp. 250-252.

Renewal of an Agreement When a Plant Closes Down, (no 1), pp. 100-101.

Renouvellement de convention, Liquidation des affaires et, (no 1), pp. 101-102.

Sécurité et légalité de l'arrêt de travail, Menace d̀ la, (no 2), pp. 255-259.

Seniority - Nature and Scope of Discretion for a Company in Applying a Seniority Clause, (no 4), pp. 481-485.

Violation of a Collective Agreement, Disciplinary Measures Where Exists an Alleged, (no 3), pp. 344-346.

Working Force, Defined, Reduction of the, (no 2), pp. 250-252. 


\section{RECENSIONS}

Backman, Jules, Wage Determination, (no 1), p. 127. - Bloom, C.F., Northrup, H.B., Economics of Labor Relations (no 4), pp. 513-514. - Brown, Ivor, Gaitskell Hugh, Bailey K. Stephen, Worswick, G.N.D., Essays in Labour History, (no 4), p. 508. - Bullock, Paul, Standards of Wage Determination, (no 1), pp. 126-127. - Bureau International du travail, La situation syndicale en U.R.S.S., (no 1), pp. 132-134. - Bureau International du travail, La situation syndicale aux EtatsUnis, (no 1), pp. 132-132. - Bureau International du travail, Au service de la petite industrie, (no 4), p. 510. - Bureau International du travail, Pourquoi les travailleurs abandonnent la terre: Etude Comparative, (no 1), pp. 125-126. Bureau International du travail, Stages à l'étranger, (no 4), pp. 512-513 - Cahier Leannec, P. Lethielleux, L'infirmière et son perfectionnement, (no 2), pp. 275-276. - Caloni, Pierre, Les préventeurs, (no 2), p. 273. - Canizarès, F. de Sola, La constitution de la société par actions en droit comparé, (no 2), pp. 272-273. - Chombart de Lauwe, M., La vie quotidienne des familles ouvrières, (no 3), pp. 366-367. Dahrendorf, Ralph, Class and Class Conflict in Industrial Society, (no 1), pp. 129130. - Dubin, Robert, The World of Work, Industrial Society and Human Relations, (no 2), p. 273. - Dubin Robert, Working Union-Management Relations, (no 2), pp. 271-272. - Dubois, Alexandre, Confidences d'un patron sur la réforme de l'entreprise, (no 1), pp. 123-124. - Galenson, Walter, Trade Union Democracy in Western Europe, (no 4), pp. 507-508. - Groupe Lyonnais d'Etudes médicales, philosophiques et biologiques, La vieillesse, problème d'aujourd'hui, (no 4), p. 511. - Harbison, Frederick \& Myers, Charles A., Management in the Industrial World, (no 4), p. 509. - Harrington, Michael and Jacobs, Paul, Labor in a Free Society, )no 1(, pp. 130-131. - Hugh-Jones, E.M., Human Relations and Modern Management, (no 2), pp. 269-270. - Industrial Relations Center, McGill University, Montreal, Union and the Future, (no 1), pp. 124-125. - Kerr Clark, Harbison Frederick, Dunlop John T., Myers Charles A., Industrialism and Industrial Man, (no 4), pp. 510-511. - Knowles, William S., Trade Union Development and Industrial Relations in the British West Indies, (no 4), p. 515. - Mann, Floyd C., and Hoffman, L. Richard, Automation and the Worker: A Study of Social Change in Power Plants, (no 1), pp. 128-129. - Meyers, Frederic, European Coal Mining Union: Structure and Function, (no 3), pp. 364-366. - Michel, Andrée, Famille, industrialisation, logement, (no 3), p. 363. - Organisation internationale du travail, Le statut et le rôle des représentants des travailleurs, (no 2), p. 274. - Piraux, Maurice, Les cadres du secteur privé en Belgique, (no 4), pp. 509-510. - Revue Esprit, La femme au travail, (no 4), p. 510. - Spyropoulous, Georges, Le droit des conventions collectives de travail dans les pays de la Communauté européenne du charbon et de l'acier, (no 2), pp. 270-271. - United States Department of Labor, Collective Bargaining in the Basic Steel Industry, (no 3), pp. 362-363. - Whyte, William Foote, Man at Work, (no 4), pp. 514-515. - Wilcock, Richard C., Sober, Irwin, Small City Job Markets: The Labor Market Behavior of Firms and Workers, (no 2), p. 275. - Zweig, Ferdynand, The Israeli Worker, (no 2), p. 276. 